\title{
Damage threshold determination of bulk polymer samples using pulsed photothermal deflection technique
}

\author{
K RAJASREE, A V RAVIKUMAR, P RADHAKRISHNAN, \\ V P N NAMPOORI and C P G VALLABHAN \\ Department of Physics, Cochin University of Science and Tecthotogy, Cochin 682022. India \\ MS receiped 17 June 1991; revised 4 January 1992
}

Abstract. Photothermal dellection technique was used for detemining the laser damage threshold of polymer samples of teflon (PTFE) and nylon. The experiment was conducted using a Q-switched Nd-YAG laser operating at its fundamental wavelength $(1.06 \mu \mathrm{m}$, pulse width $10 \mathrm{nS}$ FWHM) as irradiution source and a $\mathrm{He}$-Ne laser as the probe beam, along with a position sensitive detector. The damage threshold values determined by photothermal deflection method were in good agreement with those determined by other methods.

Keywords. Photothermal deflection technique; damage threshold; polyner, Nd-YAG laser; He- $\mathrm{Ne}$ laser.

\section{Introduction}

Damage threshold studies of polymers have gained considerable significance in recent years because of their applications in photolithography (Dyer and Sidhu 1985; Srinivasan et al 1986; Sell et al 1989; Srinivasan and Braren 1989), in the choice of optical components in laser systerns (Milam 1977; Dyumaev et al 1983) and in the selection of polymer-based nonlinear optical elements (Lipscomb et al 1981). There are various methods like surface morphological studies, visual observation of plasma emission from the target and reflectivity variation studies from the target to evaluate the laser-induced damage threshold of materials. Techniques based on photoacoustic effect have proved to be very effective in determining the laser damage thresholds of both transparent and opaque samples (Roscencwaig and Willis 1980). The present paper describes the use of transverse photothermal deftection technique (TPTD) to evaluate damage threshold of bulk polymer samples of tefion (PTFE) and nylon.

\section{Photothermal deflection process}

Absorption of laser radiation (pump beam) by a sample surface generated heat due to various non-radiative de-excitation processes occurring in the sample. The heat thus generated was transferred to the surrounding medium in close vicinity of the irradiated surface resulting in a temperature increase of the former. This inerease in temperature led to density variations which brought about a refractive index gradient in the tnedium adjacent to the surface. A probe laser beam propagating through this refractive index gradient perpendicular to the direction of the pump beam suffered refraction and consequently deviated from its original path (figure 1) corresponding to ambient condition. The effect was termed as transverse photothermal deflection (TP'TD). The magnitude of the beam deflection depended on the amount of heat transferred from the sample to the medium. Such heat transfer depended strongly on 


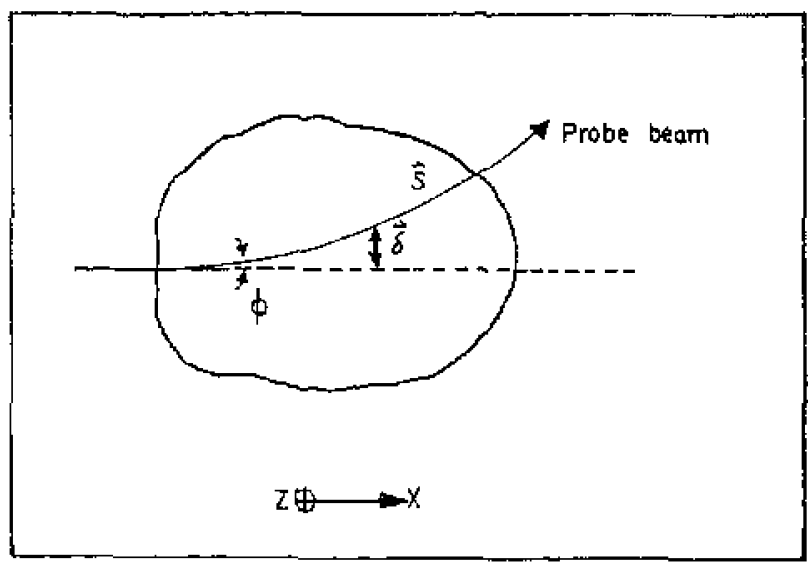

Figure 1. Deflection of the probe beam propagating in an inhomogeneous medjum duc to mirage ellect.

the thermal processes induced on the surface by laser beam and generally it was lound to increase for pump energies above the optical breakdown. As a result, at laser fluences above the damage threshold a noticeable enhancement in PTD was found.

The propagation of an optical beam (probel in an inhomogeneous medium was governed by the equation

$$
\mathrm{d} / \mathrm{d} s\left(n_{0} \mathrm{~d} \delta / \mathrm{d} s\right)=n(r, t)
$$

where $s$ was the beam path, $\delta$ the perpendicular displacement from the original path which depend on the angle of deflection $\phi$ and the position of the detector and $\boldsymbol{n}(r, t)$ the gradient of refractive index perpendicular to the beam path. If $n_{0}, T_{A}$ and $\rho_{A}$ were the refractive index, temperature and density respectively under ambient conditions, the perturbed refractive index was written as

and

$$
n(r, t)=n_{0}+\partial n /\left.\partial t\right|_{T_{A}} T(r, t)+\hat{\partial} n /\left.\partial \rho\right|_{\rho_{A}} \rho(r, t)
$$

$$
\mathrm{d} \delta / \mathrm{d} s=1 / n_{0} \partial n / \partial T \int_{\mathrm{path}} \nabla_{\perp} T(r, t) \mathrm{d} s .
$$

For small deflections we can write $\phi(x, y, t)=\mathrm{d} \delta / \mathrm{d} S$ where $\phi$ is the angle of defiection Therefore (3) may be written as

$$
\phi(x, t)=1 / n_{0} \partial n / \partial T \int \partial T / \partial x(x, y, t) \mathrm{d} y
$$

where $T(x, y, t)$ was the temperature distribution created by the heated sample surface The details of solving (4) were given by Tam (1986) and Rose and Gupta (1986). Since the probe beam profile was gaussian, the beam deflection was measured using a position sensitive detector (PSD).

To compute the thermal energy of a heated region by processing a detcctor signal one has to correlate the temperature distribution of the investigated region with the optical beam propagation through the adjacent non-homogeneous medjum and the detector response. The theoretical calculation of the probe beam deflection was verified using quadrant detector as the PSD (Jackson et al 1980). In the present investigation 


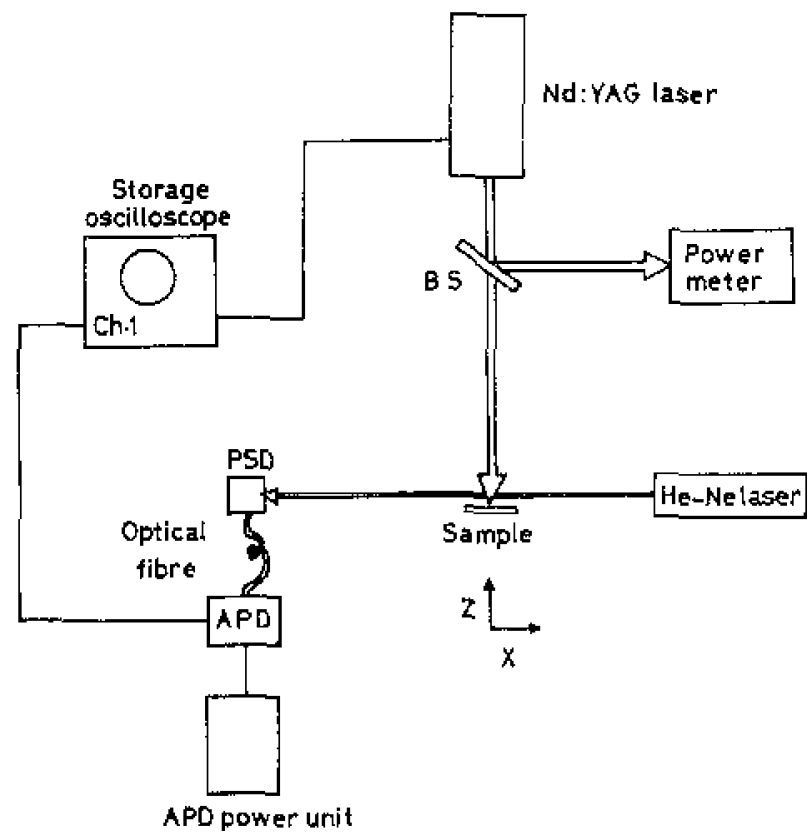

Figure 2. Schematic diagram of the experimental set-up.

the polished tip of an optical fibre coupled 10 an avalanche photodiode (APD) acts as the position sensitive detector (Rajasree et al 1990).

\section{Experimental technique}

The schematic diagram of the experimental set-up for the determination of the damage threshold of the polymer samples using the PTD technique is given in figure 2 .

The sample in the shape of a disc of $205 \mathrm{~cm}$ dia and $0.55 \mathrm{~cm}$ thickness was mounted on a micropositional XYZ translator. The pump beam used to irradiate the surface was $1.06 \mu \mathrm{m}$ radiation from a Q-switched Nd-YAG laser (Quanta ray DCR11). A short focal length convex lens focussed the pump laser beam on to the sample surface. The laser fluence incident on the sample surface was varied by adjusting the position of the lens in front of the sample. A $5 \mathrm{~mW}$ He-Ne laser beam passing parallel to and grazing the sample surface was used as the probe beam. A libre optical sensor which acts as the position sensitive detector (PSD) (Rajasree et al 1990) located at about $50 \mathrm{~cm}$ away from the sample measured the magnitude of the probe beam deflection. A $100 \mathrm{MHz}$ storage oscilloscope (Tektronix 466) coupled to the PSD recorded the transient deflection. The laser energy was monitored for each pulse using a pulsed energy meter (Deita developments) triggered in synchronization with the laser pulse.

\section{Results and discussion}

A typical oscilloscope trace of the PTD signal recorded for nylon is shown in figure 3. The peak-1o-peak value of the signal was taken as the signal amplitude. Damage threshold values evaluated for nylon and teflon using PTD tochnique are given in 

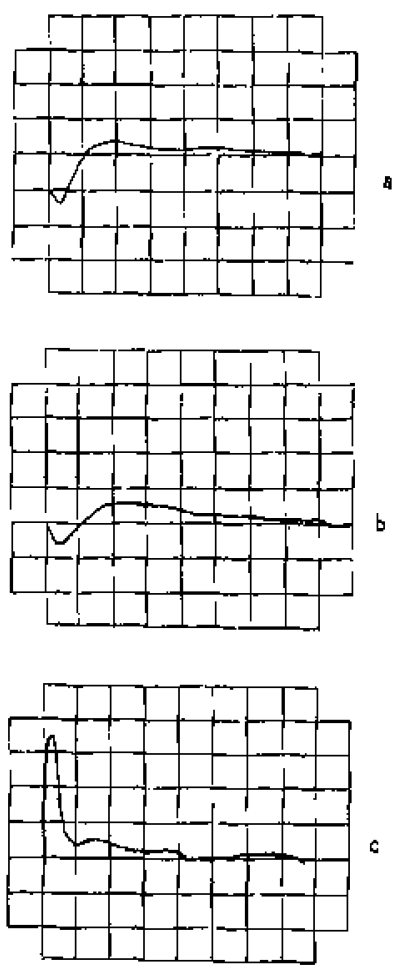

Figure 3. Oscilioseope trace of the signal from teflon at (a) $1.884 \mathrm{m.J}(0.2 \mathrm{~V} / \mathrm{div.4} 1 \mathrm{~ms}$, div. $)$ (b) $2.3 \mathrm{mj}(0.1 \mathrm{~V} / \mathrm{div} ; 1 \mathrm{~ms} / \mathrm{div})$ and (c) $3.35 \mathrm{~m})(0.1 \mathrm{~V} / \mathrm{div} ; \mathrm{l} / \mathrm{ms} / \mathrm{div}$ ).

Table 1. Energy densities at regions $A$ and $B$ for nylon and teflon.

\begin{tabular}{|c|c|c|c|c|}
\hline \multirow[b]{2}{*}{ Sample } & \multicolumn{2}{|c|}{$\begin{array}{c}\text { Region }(A) \\
\mathrm{J} / \mathrm{cm}^{2}\end{array}$} & \multicolumn{2}{|c|}{$\begin{array}{c}\text { Region (B) } \\
\mathrm{J} / \mathrm{cm}^{2}\end{array}$} \\
\hline & $\begin{array}{l}\text { Present } \\
\text { method }\end{array}$ & $\begin{array}{l}\text { Allersate } \\
\text { method* }\end{array}$ & $\begin{array}{l}\text { Present } \\
\text { method }\end{array}$ & $\begin{array}{l}\text { Alternate } \\
\text { method }\end{array}$ \\
\hline Nylon & $1 \cdot 8$ & 1.53 & $2+5$ & $2+25$ \\
\hline Teflon & 22 & 1.78 & 325 & 2.85 \\
\hline
\end{tabular}

Estimated Errot $\sim 20 \%$ * *avi Kumar et al (1991)

table 1. For comparison the results obtained with alternate methods (Ravi Kumar et at 1991) are also included here. These values showed close agreement with the results obtained from the PTD measurement.

Figures 4 and 5 show the plot of the measured signal amplitude against the energy density of the pump beam incident on the sample and these graphs exhibit two distinct regions of different slopes corresponding to two different kinds of thresholds for the laser-induced surface damage (Harada et al 1989; Ravi Kumar et al 1991). An abrupt increase in signal amplitude was found to occur in the regions near the threshold in agrement with earlier observations (Roscencwaig and Willis 1980; Srinivasan and 


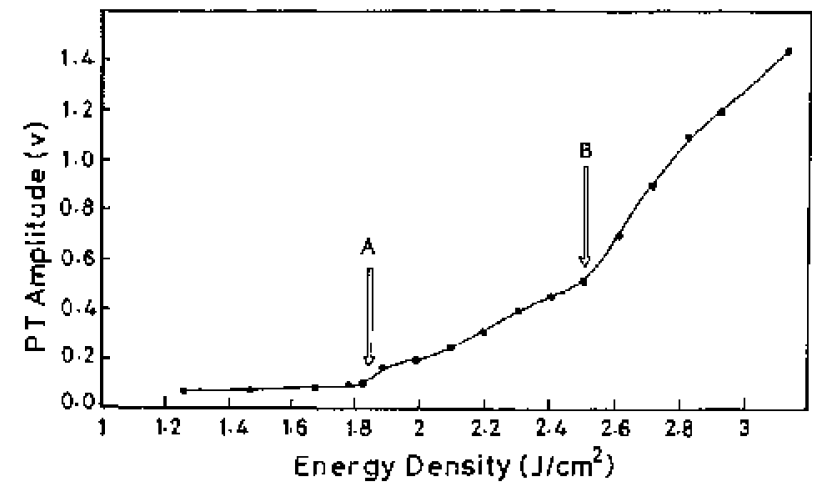

Figure 4. Plot of laser energy density ws PT amplitude for nylon in air.

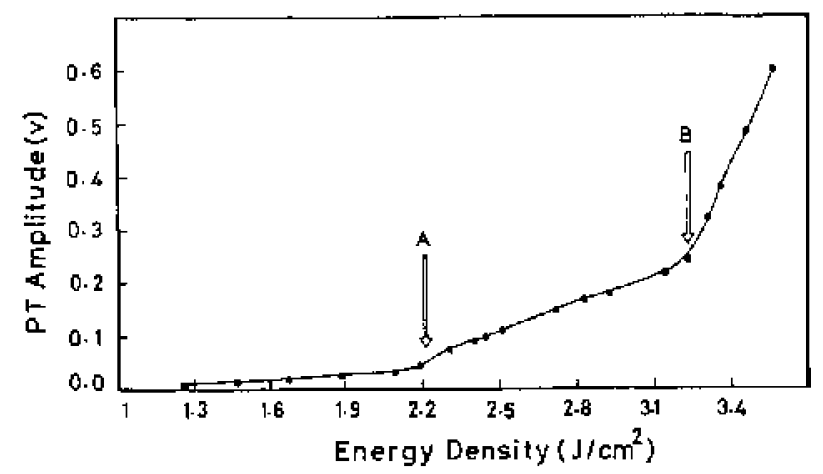

Figure S. Plot of laser energy density va PT amplitude for tellon in air.

Braren 1989; Ravi Kumar et al 1991). The two distinct threshold values explained in terms of different mechanisms operative in producing the damage-like impurity initiated damage, bond breaking and melting processes took place at different laser energy densities.

Although the mechanism of laser-induced damage in polymeric materials is not well understood, it has been observed that the damage is very sensitive to sample surface condition. The dependence of damage threshold on possible absorptive inciusions and surface polishing of the sample was earlier reported (Golberg et al 1983). Results showed that polymers with rough or opaque surfaces had lower values for damage threshold than those for the same material having polished or transparent surfaces at the same wavelength. Therefore, of the two distinct regions observed, the first region (A) corresponded to the damage due to inclusions, impurities and surface inhomogeneity, while the second (B) occurring at higher fluences were assigned to initiation of bond breaking process in the sample. It must be mentioned here that in the second case (B), in addition to the surface layer, the bulk of the material immediately below the surface also got affected in a substantial way due to the action of the laser pulse. 


\section{Conclusions}

The suitability of PTD technique to estimate the damage threshold of a polymer sample has been illustrated. Damage threshold values cvaluated by this method for nylon and teflon are in agreement with previous results but tend to be higher.

\section{Acknowledgements}

Authors are thankful to the Council of Scientific and Industrial Research, the Department of Science and Technology and the Ministry of Human Resource Development, New Delhi for financial assistance. One of the authors (RK) is gratefu1 to the University Grants Commission for a research fellowship.

\section{References}

Dyumes K M, Manentov A A, Maslyukov A P, Matyushin G A, Nechitaito v $\mathrm{S}$ and Prokhorov A M 1983 Sow. J. Quantum Electron. 13503

Dyer P E aud Sidhu J 1985 J. Appl. Phys. 571420

Golberg S M. Matyushin G A. Pilipelsky N F, Yu Savanin S, Sudarkin A N and Triblesky M I 1983 Appl. Phys. B31 85

Harada Y, Kanemitsu Y, Tunaka Y, Nakano N, Kuroda N and Yamanaka K 1989 J. Phys. D22 569

Jackson W B, Amer N M, Boccara A C and Fournier D 1980 Appl. Opt. 201333

Lipscomb G F, Grito A F and Narang P S $1981 \mathrm{~J}$. Chem, Phys. 751509

Milam D 1977 Appl. Opt. 161204

Rajaşree K, Ravi Kumar A W, Radhakrishnad P, Nampoori V P N and Valabhan C P G 1990 d. Acoustic Soc. India 1824

Ravi Kumar A V, Padmaja G, Radhakrishnan P, Nampoori V P N and Vallabhan C P G 1991 Pramona-J. Plys. 37345

Roseencwaig A and Willis J B 1980 Appl. Phys. Lett. 36.667

Rose A and Gupta R 1986 Opt. Commun. 56303

Sell J A, Heffellinger D M, Ventzek P and Gilgenbach R M 1999 Appl. Phys, Lett. 552435

Srinivasan V, Smertic M A and Babu S V 1986 J. Appl. Phys. 593681

Srinivasan R and Braren B 1989 Chem. Rev. 891303

Tam A C 1986 Rev. Mod. Phys, $\mathbf{5 8} 381$ 